\title{
Capacidad antioxidante de cinco cultivares de mango (Mangifera indica L.) y evaluación de su comportamiento en una matriz alimentaria
}

\section{The antioxidant capacity of five mango cultivars (Mangifera indica L.) and evaluation of its performance in a food matrix}
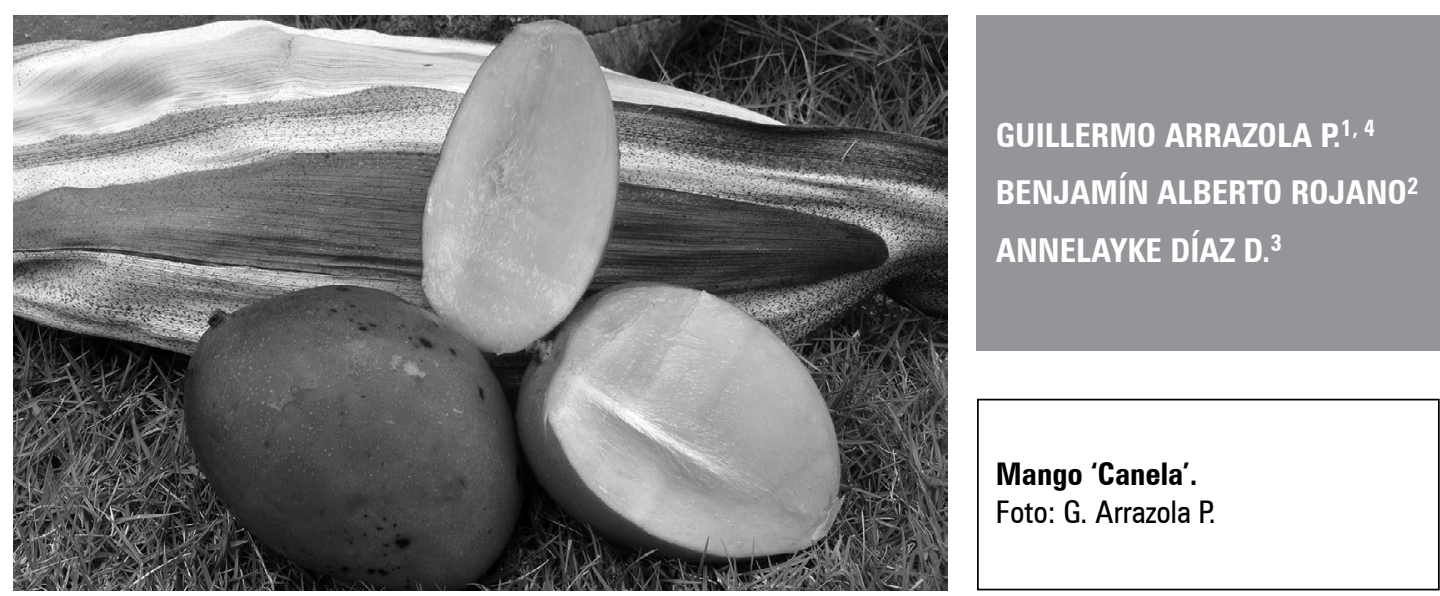

\section{RESUMEN}

La capacidad antioxidante de los extractos de cinco cultivares de mango (Corazón, Paloma, Magdalena River, Canela y Jobo) fue estudiada mediante la determinación del contenido de $\beta$-caroteno, fenoles, ácido ascórbico y la capacidad captadora de radicales libres, 2,2-difenil-1-picrylhydrazyl (DPPH). Además, se evaluó la capacidad antioxidante de los cultivares en una matriz lipídica, en comparación con un antioxidante comercial butilhidroxianisol (BHA). El IC-50 de la capacidad captadora de radicales libres para variedad Jobo fue de 0,776 el mejor resultado aplicando modelo de regresión simple $\left(R^{2}=99,58\right)$; esta variedad presentó mayor actividad antioxidante con concentración de extracto $70,35 \mathrm{mg} \mathrm{mL}^{-1}$, resultado verificado con un contenido de fenol de $208.804 \mathrm{mg} \mathrm{L}^{-1}$ de ácido gálico. Además, 'Jobo' presentó la mayor concentración de ácido ascórbico con $9.730,07 \mathrm{mg} \mathrm{kg}^{-1}$, mientras que la variedad Magdalena River mostró el más alto porcentaje de inhibición de decoloración de $\beta$-caroteno (46,53\%). Con 'Jobo' se obtuvo el mejor comportamiento en la matriz alimentaria usando $1.000 \mathrm{mg} \mathrm{kg}^{-1}$ de extracto logrando el mismo efecto del BHA por encima de la concentración permitida $\left(175 \mathrm{mg} \mathrm{kg}^{-1}\right)$.

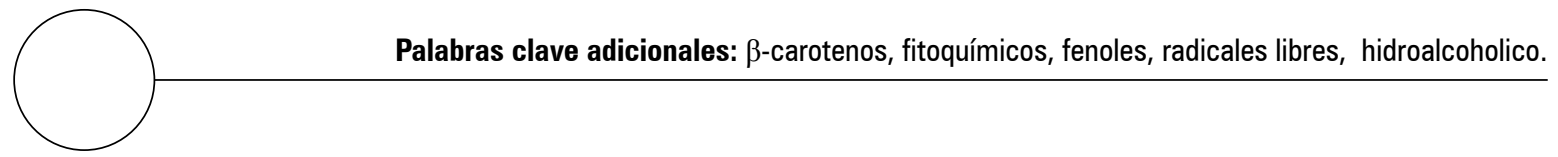

1 Facultad de Ingeniería, Departamento de Procesos Agroindustriales, Programa Ingeniería de Alimentos, Universidad de Córdoba (Colombia).

2 Facultad Ciencias, Programa de Química, Universidad Nacional de Colombia, Medellín (Colombia).

Facultad de Ciencias Agrícolas, Universidad de Córdoba (Colombia).

4 Autor para correspondencia. guillermo.arrazola@ua.es 


\section{ABSTRACT}

The antioxidant capacity of five Mangifera indica cultivar extracts (Heart, Dove, Magdalena River, Cinnamon and Jobo) was studied by determining the $\beta$-carotene content, phenols, ascorbic acid and free radical scavenging capacity of 2,2-difenil-1-picrylhydrazyl (DPPH). We assessed the antioxidant capacity of the cultivars in a lipid matrix, comparing it with that of the commercial antioxidant butylhydroxyanisole (BHA). The IC-50 of the free radical scavenging capacity for the Jobo variety was 0.776 ; the best result using a simple regression model $\left(R^{2}=99.58\right)$. This variety showed a higher antioxidant activity with an extract concentration of 70.35 $\mathrm{mg} \mathrm{mL}^{-1}$, verified by a phenol content of $208.80 \mathrm{mg} \mathrm{L}^{-1}$ of gallic acid. Furthermore, the Jobo variety had the highest concentration of ascorbic acid with $9,730.07 \mathrm{mg} \mathrm{kg}^{-1}$, while the Magdalena River variety showed the highest percentage of $\beta$-carotene discoloration inhibition (46.53\%). The Jobo variety provided the best performance in the food matrix using $1,000 \mathrm{mg} \mathrm{kg}^{-1}$ of extract, achieving the same effect of BHA over the allowable concentration $\left(175 \mathrm{mg} \mathrm{kg}^{-1}\right)$.

Additional key words: $\beta$-carotene, phytochemicals, phenols, free radicals, hydroalcoholic.

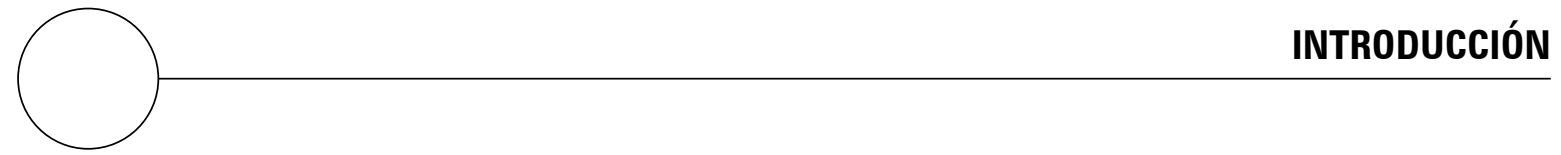

El consumo de frutas no es simplemente una consecuencia de gustos y preferencias personales, en la actualidad ayuda a solucionar problemas de salud debido al contenido de nutrientes. Además de los nutrientes esenciales, las frutas presentan compuestos antioxidantes, micronutrientes, minerales, fibras, vitaminas y compuestos fenólicos secundarios. Cada vez más pruebas demuestran la importancia de estos micronutrientes para la salud humana (Vasco y Kamal 2008; PedrazaChaverri et al., 2008).

Los antioxidantes pueden ser naturales o sintéticos y están ampliamente distribuidos en los alimentos frescos o procesados, siendo las mayores fuentes los alimentos de origen vegetal y sus productos, por presentar un alto contenido de vitamina $\mathrm{E}$, vitamina $\mathrm{C}$, carotenoides y compuestos fenólicos, específicamente flavonoides (Ercigli et al., 2008; Stipanuk, 2000; Sokol, 1997; Rodríguez, 1997). Las sustancias oxidativas presentes en los alimentos pueden atacar biomoléculas insaturadas produciendo daños y modifica- ciones químicas indeseables, siendo las de mayor frecuencia los daños a la estructura del DNA y la peroxidación lipídica de membranas celulares (Alvis et al., 2012; Olaya et al., 2009; Rojano et al., 2007). La mayoría de estos compuestos han sido estudiados como parte de la composición química y nutricional o como sustancias aisladas, por ejemplo, la acción de la vitamina E como reductor de perturbaciones oxidativas (Martínez et al., 2012). Pero una mezcla de antioxidantes, en una sola matriz como ocurre en alimentos, ha sido poco reportada (Rincón et al., 2011; Kriengsak et al., 2006). Además según estudios de algunos vegetales (Kriengsak et al., 2006; Kuskoski et al., 2005), frutos como mango, guayaba y acerola poseen propiedades medicinales, las cuales son atribuidos principalmente al conjunto de compuestos fenólicos contenidos. Palafox-Carlos et al. (2012), identificaron y cuantificaron compuestos fenólicos en mangos cultivar Ataulfo (México).

El objetivo del trabajo fue estudiar la capacidad antioxidante de cinco cultivares de mango 
(Mangifera indica L.) y la evaluación de su comportamiento en una matriz alimentaria.

\section{MATERIALES Y MÉTODOS}

La investigación se realizó en la Planta Piloto de Vegetales de la Universidad de Córdoba, municipio de Ciénaga de Oro y en el Laboratorio de Química de Alimentos, Universidad Nacional de Colombia, Medellín.

Material vegetal: el material de mango (Mangifera indica L.) fue recolectado en Montería (Sinú medio y alto) cosechando los cultivares Jobo, Canela, Corazón, Paloma y Magdalena River. Se tomaron muestras al azar en estado de madurez 3, según NTC 5139 (Icontec, 2002) y NTC 5210 (Icontec, 2003).

Lavado: se llevó a cabo por inmersión en agua potable con germicida (Mertec) durante cinco minutos.

Despulpado: se realizó una separación de pulpa, corteza y semilla. La pulpa fue homogenizada en una licuadora Oster para facilitar extracción del jugo. Para evaluar la capacidad antioxidante se realizó el siguiente tratamiento: se tomaron $4 \mathrm{~g}$ del extracto preparado, se añadieron 4 $\mathrm{mL}$ de etanol absoluto homogenizándose en un equipo Vortex Electrolab 305-30w, se centrifugó a $5.000 \mathrm{rpm} / 10 \mathrm{~min}$, en una centrífuga Sigma 2-16K, luego el sobrenadante se filtró con papel calibre 2. El filtrado se llevó a envases para protegerlos de la luz solar y poder analizarlos y utilizarlo como posible antioxidante.

Determinación de sólidos solubles totales (SST, ${ }^{\circ}$ Brix): según método 932.12/90 de la AOAC (1990) con corrección de temperatura (Icontec, 2002), macerando la pulpa de cada mango, que fue filtrado para medir el contenido de SST con refractómetro en una escala de $0-30 \%$ y corrección por temperatura y aci- dez, de acuerdo con la relación: SST corregido $=0,194^{*} \mathrm{~A}+\mathrm{SST}$, donde A es el porcentaje de ácido ascórbico y SST son los sólidos solubles totales.

Determinación de acidez total titulable: método 942.15/90 de la AOAC (1990) y NTC-4623 de Icontec (1999). El resultado se expresó como porcentaje de ácido cítrico, para efectos de cálculo se utilizó la fórmula:

$\%$ acidez titulable $=\{(\mathrm{V} 1 * \mathrm{~N}) / \mathrm{V} 2\} * \mathrm{~K}^{*} 100$

Donde, $\mathrm{V} 1$ = volumen de $\mathrm{NaOH}$ consumido $(\mathrm{mL}), \mathrm{V} 2=$ volumen de la muestra $(10 \mathrm{~mL})$, $\mathrm{K}=$ peso equivalente ácido cítrico $\left(0,064 \mathrm{~g} \mathrm{meq}^{-}\right.$ 1) $\mathrm{N}=$ normalidad del $\mathrm{NaOH}\left(0,1\right.$ meq $\left.\mathrm{mL}^{-1}\right)$.

Determinación de $\boldsymbol{p H}$ : según el método 981.12/90 de la AOAC (1990).

Determinación de humedad: método 7.003/84, 930.15/90 de la A.O.A.C adaptado (AOAC, 1990). Se determinó el contenido de humedad con un horno de circulación forzada marca Thermolab Dies a $105^{\circ} \mathrm{C}$ hasta peso constante.

Determinación de contenido total de $\beta$-caroteno: Se diluyó el radical coloreado ABTS (2,2 azinobis-(3-etilbenzotiazolin 6-ácido sulfónico) en buffer PBS, agregar $2 \mathrm{~mL}$ de ABTS concentrado a $200 \mathrm{~mL}$ de buffer PBS (persulfato de potasio). La solución se tornara verde-azul. Se midió la absorbancia de ABTS diluido en buffer PBS a 734 $\mathrm{nm}$. Se aforó a $5 \mathrm{~mL}$ incubándose a temperatura ambiente en la oscuridad por $16 \mathrm{~h}$.

Según el método de inhibición de decoloración del $\beta$-caroteno (Velioglu et al., 1998), el resultado se expresó en porcentaje de inhibición empleando la siguiente fórmula:

$A A=100\left[1 \frac{A_{0}-A_{1}}{A_{00}-A_{0 \mathrm{t}}}\right]$ 
Donde, $\mathrm{AA}=$ porcentaje de inhibición de $\beta$-caroteno; $A_{0}=$ absorbancia de la emulsión con el extracto en el tiempo cero; $\mathrm{A}_{1}=$ absorbancia de la emulsión con el extracto en el tiempo $t ; \mathrm{A}_{0 t}=$ absorbancia de la emulsión sin el extracto en el tiempo t; $\mathrm{A}_{00}=$ absorbancia de la emulsión sin el extracto en el tiempo cero.

Contenido de ácido ascórbico: método 43.064068 de la AOAC (1990). El resultado se expresó en mg kg-1 ácido ascórbico; se realizó una curva estándar con datos experimentales aplicando un modelo de regresión lineal usando el software estadístico Statgraphics plus versión 2.0.

Cálculo de la capacidad captadora de radicales libres: método de la capacidad captadora de radicales libres DPPH (Brand et al., 1995; Espin et al., 2000) adaptado. Para efectos de cálculo se empleó la siguiente fórmula:

$A A=100\left[1 \frac{A_{0}-A_{1}}{A_{00}-A_{0 \mathrm{t}}}\right]$

Donde, $\mathrm{AA}=$ porcentaje de inhibición de radicales libre DPPH; $\mathrm{A}_{0}=$ absorbancia de la solución con el extracto en el tiempo cero; $A_{1}=$ absorbancia de la solución con el extracto en el tiempo t; $\mathrm{A}_{0 \mathrm{t}}=$ absorbancia de la solución sin el extracto en el tiempo t; $A_{00}=$ absorbancia de la solución sin el extracto en el tiempo cero.

Para obtener el IC-50 que es la concentración a la cual se inhibe el 50\% del radical, se utilizó la ecuación que mejor se ajustó al modelo de regresión aplicado a cada variedad. Para ajustar el modelo de regresión los datos fueron analizados en el software estadístico Statgraphics plus versión 2.0.

Contenido de fenoles: según el método FolinCiocalteu modificado (Singleton y Rossi, 1965), los resultados fueron expresados en $\mathrm{mg} \mathrm{L}^{-1}$ de ácido gálico, los cálculos se realizaron mediante una curva estándar método patrón externo, a partir de datos experimentales a los cuales se les aplicó un modelo de regresión lineal mediante el software estadístico Statgraphics plus versión 2.0 .

Evaluación de la capacidad antioxidante en un matriz lipídica: método de determinación del índice de peróxidos (Kirk et al., 2003), los resultados fueron expresados meq-g $\mathrm{kg}^{-1}$, para efectos de cálculo se empleó la fórmula:

$I P=\frac{(\mathrm{V})\left(\mathrm{Na}_{2} \mathrm{SO}_{3} \mathrm{O}_{3}(100)\right.}{M}$

Donde, IP = índice de peróxidos; $\mathrm{V}=$ volumen gastado de tiosulfato; $M=$ masa de la muestra (g); $\mathrm{Na}_{2} \mathrm{SO}_{3} \mathrm{O}_{3}=\operatorname{normalidad}(0,0096 \mathrm{~N})$.

La matriz lipídica utilizada fue mantequilla ya que tiene alto contenido de ácidos grasos como el palmítico y esteárico con 16 y 18 dobles enlaces de carbono, por ser ácidos grasos provenientes de rumiantes están combinados entre sí en 45$50 \%$. Para este tipo de alimentos se hace necesario hacer un control de calidad estricto a nivel industrial ya que son sensibles a la oxidación que es catalizada por las altas temperaturas y exposición a la luz; el fenómeno de oxidación para este tipo de matrices es conocido como "rancidez" (Garret, 1993; Castaldo, 1998). Para acelerar el proceso de oxidación en las muestras analizadas fue necesario dejarlas a temperatura ambiente y expuestas al ambiente por un periodo de $72 \mathrm{~h}$ donde el índice de peróxidos se determinó cada ocho horas (Cifuentes, 2003).

Análisis estadístico: la capacidad antioxidante se realizó por triplicado para las variables analizadas. Se tabuló la información de las variables con un diseño completamente al azar con estructura factorial donde el primer factor fueron las cultivares de mango y el segundo factor las concentraciones del contenido de fenoles. Las variables consideradas fueron relacionadas en un análisis de varianza tipo III-SC en el que al 
factor concentración y variedad se le realizó una prueba de comparación de medias (Tukey) a un nivel de confianza del 95\%, empleando el software estadístico Statgraphics plus versión 2.0.

\section{RESULTADOS Y DISCUSIÓN}

Según NTC-5139 (Icontec, 2002), el índice de madurez de mangos criollos va relacionado con los cambios de color interno y externo y varía en una escala de 1-5, la cual se confirma con la relación entre sólidos solubles totales y la acidez titulable. Los cultivares estudiados se analizaron en estado de madurez 3 , investigaciones que han evaluado la capacidad antioxidante en frutas tropicales no definen un criterio específico para analizar las frutas en un estado de madurez; sin embargo, la literatura reporta que las frutas tropicales son buenas fuentes de antioxidantes naturales (Wang y Li, 2000; Kanazawa y Sakibara, 2000).

\section{Determinación del contenido total de $\beta$-caroteno}

Se encontraron diferencias estadísticas significativas entre cultivares. El factor concentración no mostró diferencias estadísticamente significativas entre cultivares $(\mathrm{F}=2,17<\mathrm{Ftab}$.); para el factor variedad la prueba mostró diferencias estadísticamente significativas $(P<0,05 \mathrm{y}$ $\mathrm{F}=33,49$ ); el porcentaje más alto de inhibición de $\beta$-caroteno lo mostró 'Magdalena River' con una media de $43,51 \%$ (tabla 1 ). Cada variedad tiene compuestos que la hacen diferente una de otra, aunque pertenezcan a la misma familia (Anacardiaceae). La variedad que presentó menor inhibición de decoloración de $\beta$-carotenos fue Jobo (tabla 1), esto no indica que estos compuestos no estén presentes en esta variedad, ya que la baja actividad antioxidante de una sustancia en el sistema $\beta$-caroteno da información de su capacidad protectora en sistemas lipídicos. Estudios en la variedad Kent, determinaron que los carotenos son predominantes casi en $84 \%$ de la concentración de carotenoides totales, identificando por
HPLC como carotenoides predominantes violaxanthin, neochrom, luteoxanthin y $\beta$-caroteno, aunque esto no compromete la fuerza de inhibición de radicales libres por parte de estos carotenoides (Pott et al., 2003). Similarmente, Chen et al. (2005) identificaron y cuantificaron los carotenoides presentes en mango Taiwanese encontrando que todos los trans- $\beta$-carotenos y sus cis isómeros constituyeron a la mayor proporción correspondiente a $18,55 \mu \mathrm{g} \mathrm{g}^{-1}$, seguida de neochrom con $8,36 \mu \mathrm{g} \mathrm{g}^{-1}$, violaxanthin y sus cis isómeros $2,46 \mu \mathrm{g} \mathrm{g} \mathrm{g}^{-1}$, luteoxanthin $2,42 \mu \mathrm{g}$ $\mathrm{g}^{-1}$, neoxanthin $1,55 \mu \mathrm{g} \mathrm{g}^{-1}$, presentándose en el mango 'Magdalena River' un índice de inhibición del 43,506\%, dada la cantidad de carotenoides en el fruto.

\section{Determinación del contenido de ácido ascórbico}

Los datos experimentales obtenidos fueron ajustados a un modelo de regresión lineal simple $Y=3,6225 \mathrm{X}+0,0214$, con $R^{2}$ de 0,97 . En la tabla 1 se observa que el contenido de ácido ascórbico difiere para cada uno de los cultivares estudiadas, presentando la mayor concentración el cultivar Jobo con un valor de igual (9.036,24 mg $\mathrm{kg}^{-1}$ ) y Magdalena River la menor concentración con un valor de $76,036 \mathrm{mg} \mathrm{kg}^{-1}$. El ácido ascórbico está involucrado en reacciones inmunológicas y antibacteriales y protege la membrana de leucocitos por daños de oxigenación (Madhavi, 1995). El análisis de varianza y la comparación de medias presentaron diferencias estadísticas significativas en el contenido de ácido ascórbico entre los cultivares $(P<0,05 ; \mathrm{F}=76,81)$; al evaluar el factor concentración se obtuvo que no hubo diferencias significativas entre el efecto de las concentraciones de los filtrados de cultivares $(P>0,05 ; \mathrm{F}=0,76)$. El test de Tukey mostró que hay diferencias estadísticas significativas entre 'Jobo' con el resto de cultivares incluyendo la variedad Corazón. Kondo et al. (2005) cuantificaron el contenido de ácido ascórbico en corteza y pulpa de mango del cual reportaron valores de $0,74 \mathrm{mmol} \mathrm{kg}^{-1}$ y $0,72 \mathrm{mmol} \mathrm{kg}^{-1}$ de ácido ascórbico respectivamente, determinando así un 
poder antioxidante moderado, de las variedades estudiadas Canela es quien mayor porcentaje contiene de Vitamina C o ácido ascórbico, dado su porcentaje de inhibición. El cultivar Jobo, contiene $0,009 \mathrm{mg} \mathrm{kg}^{-1}$ de pulpa, con lo cual la capacidad antioxidante es representativa.

\section{Cálculo de la capacidad captadora de radicales libres}

Se encontraron diferencias estadísticas entre los cultivares estudiados con respecto a la inhibición de radicales libres DPPH $(P<0,05 ; \mathrm{F}=40,3)$. La mayor probabilidad de inhibición de radicales libres corresponde a variedad Corazón con una media igual a $60,004 \mathrm{mg} / 100$ g muestra ( $\mathrm{F} \mathrm{Cal}=40,31)$; el test de rangos múltiples mostró la heterogeneidad de los cultivares, con medias en las variedades Paloma (50,690), Canela $(30,317)$, Jobo $(46,197)$ y Magdalena River (49,835 mg/100 g de muestra). La comparación de medias entre Corazón y los demás cultivares al igual que la variedad Canela y el resto de los cultivares presentaron diferencias significativas. En el análisis de varianza realizado para el factor concentración se apreció que las concentraciones tienen un efecto diferente en los cultivares estudiados $(P<0,05 ; \quad \mathrm{F}=186,39)$. Los resultados de los análisis muestran que para la variedad Corazón tiene la mejor disposición para inhibición de radicales libres. Suaterna (2001) reportó una actividad antioxidante mango en cultivar Azúcar de 57,27 2,46 midiendo durante 24 h. Según Brand-Williams et al. (1995), la concentración óptima para inhibir el 50\% del radical DPPH se conoce como IC-50. La relación entre el porcen- taje de inhibición de radicales libres DPPH y la concentración del extracto de cada variedad es altamente significativa para los modelos de regresión polinomial a un nivel de significancia del $\left.5 \%\left(P<0,05 ; R^{2}>0,97\right)\right)$. En la tabla 2 se presentan valores de IC-50, obtenidos de cada variedad con modelos de regresión simple que presentaron coeficientes de determinación cercanos al $100 \%$; en términos de cantidad los valores de IC-50 más bajos son los óptimos.

\section{Contenido de fenoles}

Los datos experimentales obtenidos fueron ajustados en una regresión simple lineal ( $\mathrm{Y}=0,0059 \mathrm{X}+0,0136$ con un $R^{2}$ de 0,997$)$. El análisis de media para el factor variedad presentó diferencias significativas entre los cultivares $(P<0,05 ; \mathrm{F}=55,20)$. Para el factor concentración no obtuvo diferencias estadísticas entre cultivares a una misma concentración del extracto $(P<0,05 ; \mathrm{F}=0,06)$. La mayor concentración promedio de ácido gálico fue determinada en 'Jobo' con un valor de $183.232,157 \mathrm{mg} \mathrm{kg}^{-1}$ (tabla 1). En puré de mangos se cuantificó el ácido gálico encontrándose cantidades iguales a $305 \mathrm{mg} \mathrm{kg}^{-1}$, ácido p-cumárico igual a $46 \mathrm{mg} \mathrm{kg}^{-1}$, ácido tánico igual a $413 \mathrm{mg} \mathrm{kg}^{-1}$ y ácido protocatecuhico igual a $198 \mathrm{mg} \mathrm{kg}^{-1}$, relacionando claramente la alta ocurrencia de los compuestos fenólicos como antioxidantes de los cuales el ácido tánico presentó actividad fuerte y el ácido p-cumárico, a pesar de encontrarse en cantidades grandes, presentó actividad débil. Fu et al. (2011) reportaron para el mango 'Shuisian' valores de 4,86 $\mu \mathrm{mol} F$ (II) $\mathrm{g}^{-1}$, 4,01 $\mu \mathrm{mol}$ Trolox $\mathrm{g}^{-1}, 37,03 \mathrm{mg}$ GAE/100g

Tabla 1. Inhibición de $\beta$-caroteno, ácido ascórbico y gálico.

\begin{tabular}{|l|c|c|c|}
\hline Cultivares & $\beta$-caroteno (\%) & Ácido ascórbico $\left(\mathrm{mg} \mathrm{kg}^{-1}\right)$ & Ácido gálico $\left(\mathrm{mg} \mathrm{kg}^{-1}\right)$ \\
\hline Magdalena River & 43,506 & 76,036 & $23.546,708$ \\
\hline Paloma & 25,094 & 17,858 & $57.006,598$ \\
\hline Jobo & 6,348 & $9.063,235$ & $183.232,157$ \\
\hline Canela & 38,101 & 154,654 & $6.697,995$ \\
\hline Corazón & 38,999 & 846,210 & $58.461,615$ \\
\hline
\end{tabular}


muestra. Estudios de Suaterna (2001) y Soong y Barlow (2005) cuantificaron el ácido gálico en la variedad Kernel donde se encontró un contenido de ácido gálico de 23,3 mg/100 g de muestra.

\section{Índice de peróxidos}

En esta técnica se aprovechó la propiedad que presentan los hidroperóxidos en un gran rango de moléculas, para reaccionar de forma cuantitativa y estequiométrica con el yoduro (Kirk, 2003). El extracto que presentó mayor protección del sistema fue variedad Jobo con un incremento mínimo en el índice de peróxidos a través del tiempo, su comportamiento se encontró por encima del comportamiento de la muestra con BHA; sin embargo, ambas muestras presentaron estabilidad muy parecidas, la matriz lipídica fue la mantequilla. La tabla 3 muestra el comportamiento de cada extracto en esta evaluación, obteniendo un resultado más que satisfactorio para el extracto de 'Jobo', dado que a partir de $1.000 \mathrm{mg} \mathrm{kg}^{-1}$ de este extracto se presentó casi la misma efectividad que BHA a la concentración máxima legislada $\left(175 \mathrm{mg} \mathrm{kg}^{-1}\right.$ ) según la FDA (Food and Drug Administration, Estados Unidos). Takebashi et al. (2013) muestran valores en hortalizas de 1,63-66,07 $\mu \mathrm{mol} \mathrm{TE} \mathrm{g}^{-1}$, en frutas concentraciones de 1,58-33,47 $\mu \mathrm{mol} \mathrm{TE} \mathrm{g}{ }^{-1}$, muy parecidas a las concentraciones de cultivares de mango estudiadas.

Correlación de las variables contenido de ácido ascórbico, $\beta$-caroteno y porcentaje de inhibición de radicales libres 2,2-difenil-1-picrylhydrazyl (DPPH) con el contenido de fenoles

Al correlacionar todas las variables dependientes estudiadas, se tomó como variable independiente el contenido de fenoles en los cinco cultivares con el objetivo de evaluar el grado de correlación entre las variables, porque se ha reportado que el

Tabla 2. Valores IC-50 obtenidos de cada variedad con modelos de regresión simple y polinomial de segundo grado.

\begin{tabular}{|c|c|c|c|}
\hline \multirow{2}{*}{ Cultivares } & \multicolumn{2}{|c|}{$\mathrm{IC}-50\left(\mathrm{mg} \mathrm{mL}^{-1}\right)$} & \multirow[b]{2}{*}{ Reg. polinomial } \\
\hline & Reg. simple & Modelo & \\
\hline Magdalena River & 6,523932 & $Y=A+B \ln X$ & 6,643495 \\
\hline Paloma & 13,285870 & $Y=A+B \ln X$ & 13,31607 \\
\hline Jobo & 0,775389 & $Y=A+B(X)^{1 / 2}$ & 2,342674 \\
\hline Canela & 24,395800 & $Y=A+B(X)^{1 / 2}$ & 7,671682 \\
\hline Corazón & 3,173933 & $Y=A+B(X)^{1 / 2}$ & 2,607986 \\
\hline
\end{tabular}

Tabla 3. Índice de peróxidos de cinco cultivares de mango (meq $\mathbf{0}_{2} \mathbf{~ k g}^{-1}$ muestra).

\begin{tabular}{|l|c|c|c|c|c|c|c|c|}
\hline \multirow{2}{*}{ Cultivares } & \multicolumn{9}{c|}{ Tiempo (horas) } \\
\cline { 2 - 10 } & 0 & 14,5 & 25 & 41 & 49,5 & 66 & 72 \\
\hline Magdalena River & 2,575 & 4,099 & 4,126 & 5,453 & 6,043 & 7,272 & 7,345 \\
\hline Paloma & 2,575 & 3,227 & 4,470 & 4,798 & 5,828 & 7,272 & 7,285 \\
\hline Canela & 2,719 & 3,227 & 3,427 & 4,065 & 4,746 & 5,381 & 5,380 \\
\hline Corazón & 2,719 & 3,427 & 4,199 & 4,784 & 5,810 & 6,043 & 6,845 \\
\hline Jobo & 3,665 & 3,683 & 3,995 & 4,199 & 4,296 & 4,470 & 5,470 \\
\hline BHA & 2,617 & 3,118 & 3,488 & 3,916 & 4,168 & 4,309 & 4,870 \\
\hline Referencia & 1,039 & 2,934 & 3,469 & 4,784 & 4,798 & 5,828 & 5,986 \\
\hline
\end{tabular}


contenido de fenoles son los antioxidantes con mayor incidencia en la protección de sistemas lipídicos y enzimáticos por ser antioxidantes primarios y tener la habilidad de eliminar radicales libres y acomplejar iones metálicos (Pietta, 2000; Soon y Barlow, 2004; Procházková et al., 2011). La dispersión de puntos, observada en la figura 1, demuestra el bajo grado de correlación entre las variables, sumado a esto, ninguno de los modelos de regresión simple usados se ajustó a esta relación, ya que los coeficientes de determinación describieron a estas variables en un porcentaje por debajo del 15\% siendo el mayor igual a 13,83\% para un modelo de regresión simple recíproco en la variable dependiente, indicando que no hay relación estadísticamente signi-

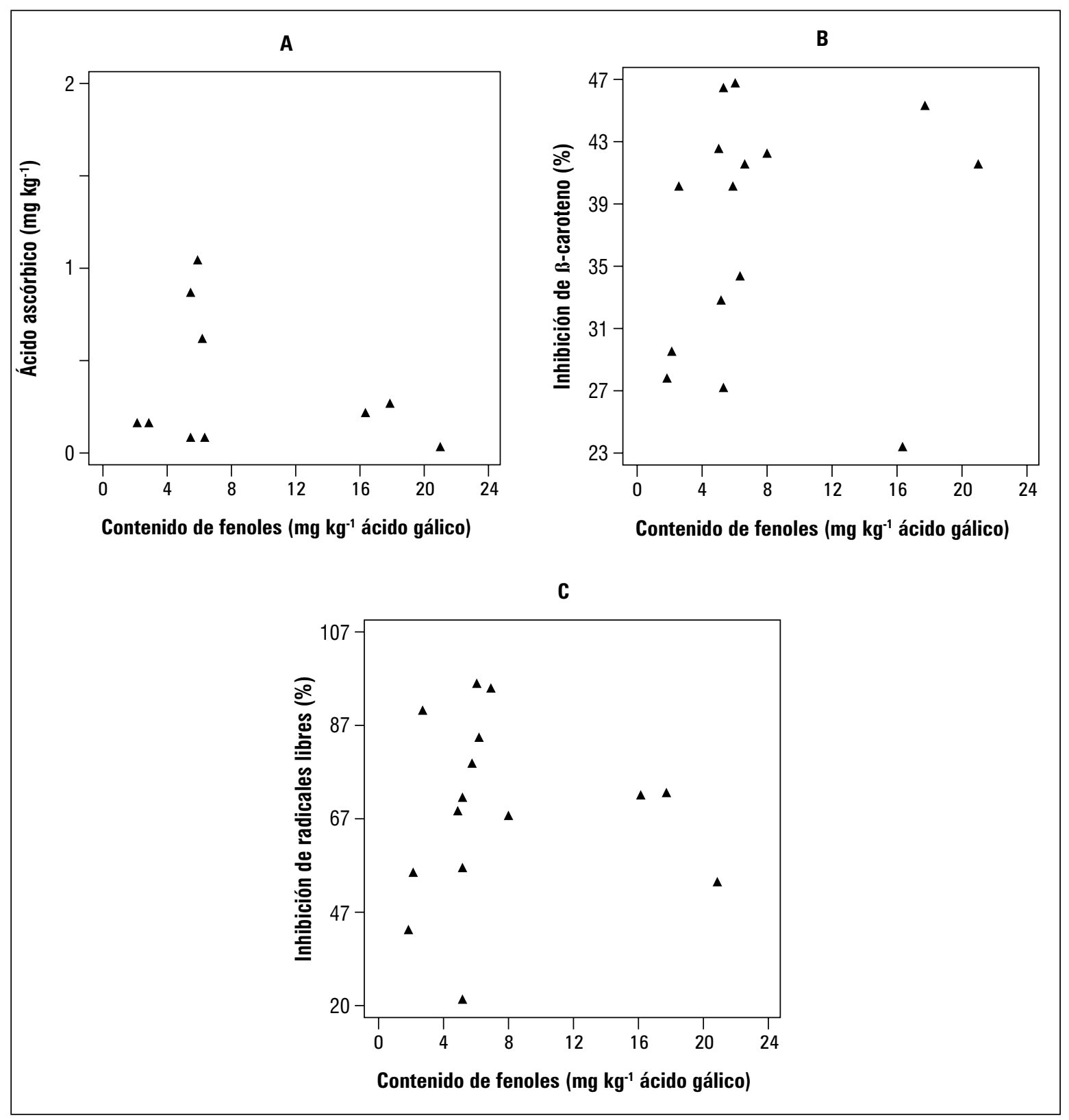

Figura 1. Correlación de las variables contenido de (A) ácido ascórbico, (B) porcentaje de inhibición de $\beta$-caroteno y (C) de radicales libres DPPH con el contenido de fenoles. 
ficativa entre estas variables $(P>0,05)$; lo que quiere decir que el contenido de ácido ascórbico, $\beta$-caroteno y la capacidad de inhibir radicales libres DPPH no dependen del contenido de fenoles en cada uno de los cultivares. Vinson (1998) revisó algunos trabajos y correlacionó el contenido de fenoles en vegetales contra la actividad antioxidante a través del método de blanqueamiento del $\beta$-caroteno, y encontró un coeficiente de correlación de $R=0,77$; de igual forma, Kalt (1999) trabajó en fresas, frambuesas y moras azules, encontrando una buena correlación entre la actividad antioxidante por el método ORAC (capacidad de absorbancia del radical oxígeno) y el contenido de fenoles. Velioglu (1998) relacionó la capacidad antioxidante en vegetales, frutas seleccionadas y productos de grano usando el método ORAC con el contenido de fenoles totales, encontrando una buena correlación $(R=0,89)$. Sin embargo, Heinonen (1998) no encontró correlación entre el contenido de fenoles totales y la actividad antioxidante por el método de oxidación del metil linoleato, entre bayas y vinos de fruta ( $R$ entre 0,32 y 0,47). Dziri et al. (2012) encontraron para diferentes partes de Alliun roseum var. Odoratissinum) contenido de flavonoides desde $26 \mathrm{mg} / 100 \mathrm{~g}$ de muestra y fenoles totales desde $29,3 \mathrm{mg} / 100 \mathrm{~g}$ de muestra.

\section{Análisis del clúster}

Este análisis se realizó con el fin de aprovechar características comunes para su agrupación (figura 2) donde las observaciones son agrupadas en un rango de distancias usando el método euclidiano. Para este caso los grupos presentaron características totalmente diferentes ya que se agruparon datos del contenido de ácido ascórbico, fenoles e inhibición de $\beta$-carotenos, sin tener relación directa entre ellos ya que cada variable es independiente de las demás y esto se pudo observar con los modelos de regresión simple que se ensayaron para cada una de las correlaciones aplicadas. Los centroides fueron calculados para cada una de las variables; los valores indican el punto en que hay relación directa entre las tres

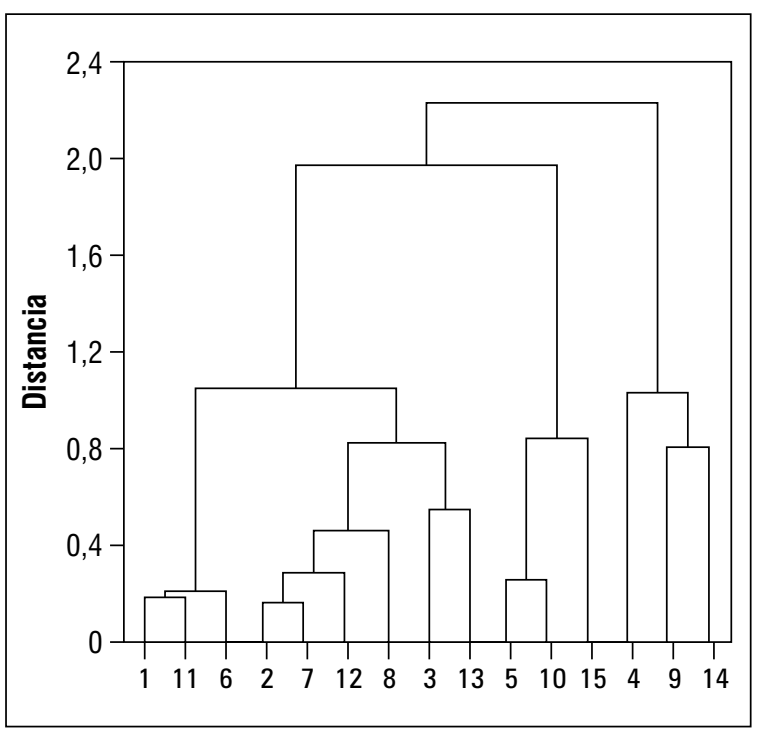

Figura 2. Dendograma del análisis de clúster. Subconjuntos respecto al contenido de características comunes para la totalidad de las variedades.

variables (figura 3) y ninguno de los datos de las variables dependientes presentó cercanía a este centroide. Este resultado también confirma que cada variedad de mango analizada tiene comportamiento diferente entre ellas con respecto a la actividad antioxidante, a pesar de pertenecer a la misma familia (Anacardiaceae).

\section{CONCLUSIONES}

Los extractos estudiados presentaron buena capacidad antioxidante; a pesar de pertenecer a la misma familia taxonómica, los cinco cultivares estudiados presentaron comportamiento diferente destacándose en la inhibición de decoloración del $\beta$-caroteno la var. Magdalena River por haber presentado el mayor porcentaje de inhibición y 'Jobo' por presentar el menor porcentaje de 13 inhibiciones. Esto va muy ligado a la coloración de los extractos sin indicar la concentración de la totalidad de los carotenoides presentes en el mango. Se encontró un contenido alto de ácido ascórbico para la variedad Jobo, esto indica que es una buena fuente de esta sustancia. Los compuestos fenólicos son los que tiene mayor inci- 


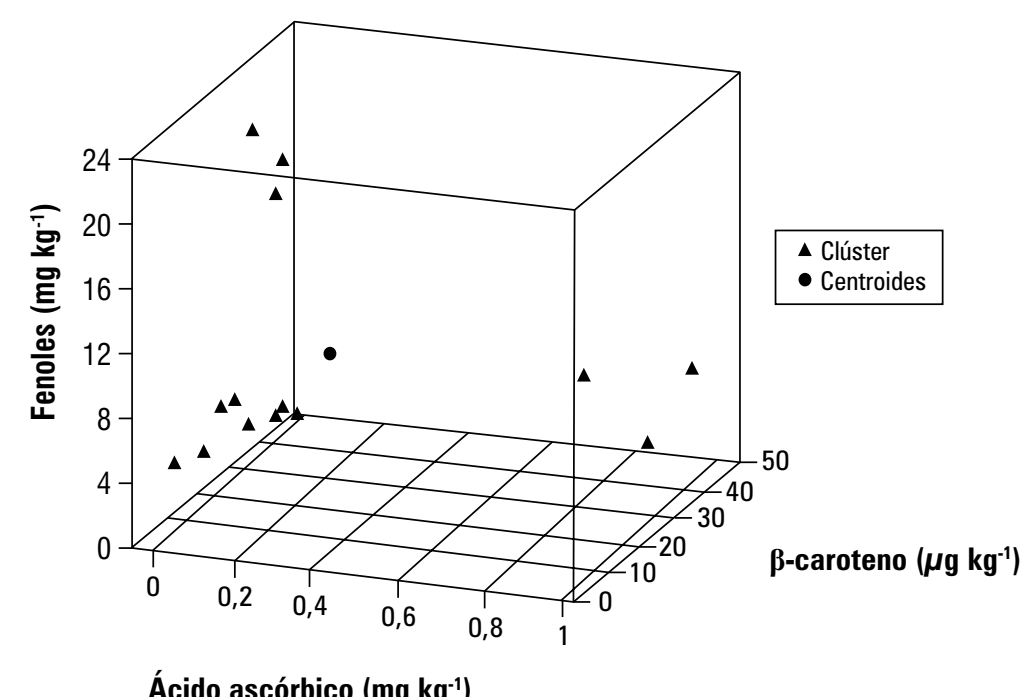

Ácido ascórbico $\left(\mathrm{mg} \mathrm{kg}^{-1}\right)$

Figura 3. Clúster 3D. Correlación de los cinco cultivares estudiadas con respecto a fenoles, ácido ascórbico y $\beta$-caroteno.

dencia en el poder antioxidante de los extractos, para esta investigación 'Jobo' fue la que presentó mayor contenido de ácido gálico, por lo que se considera una especie con alto poder antioxidante, ya que no solo se destacó para esta técnica sino que también lo hizo para inhibir radicales libres DPPH y presentar el mayor contenido de ácido ascórbico. La estabilidad de este extracto para proteger sistemas lipídicos se comprobó con el índice de peróxidos evaluado, donde la variedad Jobo mostró un comportamiento similar al antioxidante comercial BHA, por lo que se concluye que esta variedad tiene compuestos antioxidantes naturales para proteger sistemas lipídicos, lo que no descalifica a los demás cultivares que presentaron un buen comportamiento antioxidante.

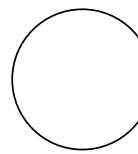

Alvis, A., G. Arrázola y W. Martínez 2012. Evaluación de la actividad y potencial antioxidante de extractos hidroalcoholicos de la Cúrcuma (Cúrcuma longa). Información Tecnológica 23(2), 11 18.

AOAC. 1990. Oficial method of analysis. Hoorwitz, N., P. Chialo y H. Reynold (eds.). $16^{\text {th }}$ ed. Association of Official Analytical Chemistry, Washington D.C.

Brands-Williams, W., M. Cuvelier y C. Berset. 1995. Use a free radical to evaluate antioxidant capacity. Lebensm.- Wiss. Technol. 28, 25-30.

\section{REFERENCIAS BIBLIOGRÁFICAS}

Castaldo, D.J. 1998. Focus on feed grade fat. Part 2 - Fat quality parameters. En: http://foodtab.com/fat2. hml; consulta: octubre de 2013.

Chen, J., C. Tai y B. Chen. 2005. Effects of different drying treatments on the stability of carotenoids in Taiwanese mango (Mangifera indica L.). J. Agric. Food Chem. 100, 1-6.

Dziri, S., I. Hassen, S. Fatnassi, Y. Mrabet, H. Casabianca, B. Hanchi y K. Hosni. 2012. Phenolic contituents, antioxidant and autimicrobial activities of Rosy garlic (Allium roseum var. odoratissinum). J. Functional Foods 4, 423-432. 
Ercigli, S., M.O. Akbulut, M. Ozdemir, E. Sengul y E. Orhan. 2008. Phenolic and antioxidant diversity among persimmon (Diospyrus kaki L) genotypes in Turkey. Int. Food Sci. Nutr. 58. 477-482.

Espin, J.C., C. Soler-Rivas y H.J. Wichers. 2000. Use to free radical dpph methods to evaluate antioxidant activity. J. Agric. Food Chem. 48, 648-656.

Fu, L., B.-T. Xu, X.-R Xu, R.-Y. Gan, Y. Zhang, E.-O. Xia y H.-B. Li. 2011. Antioxidant capacities and total phenolic contents of 62 fruits. Food Chem. 129, 345-350.

Garrett, R. 1993. Evaluating the quality of feed grade fats. pp. 2076-2082. Proc. Carolina Poultry Nutrition Conference. Charlotte, NV.

Heinonen, I., P. Lehtonen y A. Hopia, A. 1998. Antioxidant activity of berry and wines and liquors. J. Agric. Food Chem. 46, 25-31.

Icontec. 1999. NTC-4623. Productos de frutas y verduras. Determinación de la acidez titulable. Instituto Colombiano de Normas Técnicas y Especificaciones, Bogotá

Icontec. 2002. NTC-5139 para frutas frescas, mangos criollos y especificaciones. Instituto Colombiano de Normas Técnicas y Especificaciones, Bogotá.

Icontec. 2003. NTC-5210 para frutas frescas, mango, variedades mejoradas, especificaciones para cultivares mejoradas. Instituto Colombiano de Normas Técnicas y Especificaciones, Bogotá.

Kanazawa, K. y H. Sakibara. 2000. High content of dopamine, a strong antioxidant, in Cavendish banana. J. Agric. Food Chem. 48, 844-848.

Kalt, W., C.F. Forney, A. Martin y R. Prior. 1999. Antioxidant capacity, vitamin C, phenolics and anthocyanins after fresh storage of small fruit. J. Agric. Food Chem. 47, 4638-4644.

Kirk, S.R., R. Sawyer y H. Egan. 2003. Composición y análisis de alimentos de Pearson. Compañía Editorial Continental S.A. de C.V., México D.F.

Kondo, S., M. Kittikorn y S. Kanlayanarat. 2005. Preharvest antioxidant activities of tropical fruit and effect of low temperature storage on antioxidants and jamonates. Postharv. Biol. Technol. 36, 309-318.

Kriengsak, T., U. Boonprakoba, K. Crosbyb, L. Cisneros-Zevallosc y D. Hawkins. 2006. Comparison of ABTS, DPPH, FRAP, and ORAC assays for estimating antioxidant activity from guava fruit extracts. J. Food Comp. Anal. 19, 669-675.

Kuskoski, M., A. Asuero, A. Troncoso, J. Mancini-Filho y R. Fett. 2005. Aplicación de diversos métodos químicos para determinar actividad antioxidante en pulpa de frutos. Ciênc. Tecnol. Aliment. 25(4), 726-732

Madhavi, D., S. Deshpande y D. Salunke. D. 1995. Food antioxidants technological and health perspectives. Marcel Delker Inc., New York, NY.

Martínez. W., A. Alvis y G. Arrázola. 2012. Evaluación de las propiedades antioxidantes de 2 extractos de plantas aromáticas Limoncillo (Cymbopogon citratus) y Cúrcuma (Curcuma longa) y su aplicación en una matriz alimentaria. Información Tecnológica 23(2), 3-10.

Olaya, C., M. Castaño y G. Garzón. 2009. Stability of anthocyanins from Rubus glaucus Benth. and Solanum betaceum Cav. dark-red strain as affected by temperature, storage time and water activity. Acta Biol. Colomb. 14(3), 141-156.

Palafox-Carlos, E. Yahia y A. González-Aguilar. 2012. Identification and quantification of major phenolic compounds from mango (Mangifera indica, cv. Ataulfo) fruit by HPLC-DAD-MS/MS-ESI and their individual contribution to the antioxidant activity during ripening. Food Chem. 135, 105-111.

Pedraza-Chaverri, J., N. Cardenas, M. Orozco y J. Perez. 2008. Medicinal properties of mangosteen (Garcinia mangostana L.). Food Chem. Toxicol. 46, 3227 3239.

Pietta, P.G. 2000. Flavonoid as antioxidants. J. Natural Products 63(7), 1035-1042.

Pott, I., D. Breithaupt y C. Reinhold. 2003. Detection of unusual ester carotenoid in fresh mango. Mangifera indica L. cv. Kent. Phytochem. 64(4), 825-829.

Procházková, D., I. Bousova y N. Wilhelmová. 2011. Antioxidant and prooxidant properties of flavonoids. Fitoterapia 82, 513-523.

Rincón, A., D. González, L. Rached, U. Emaldi y F. Padilla. 2011. Actividad antioxidante y contenido de polifenoles en frutos de túpiro (Solanum sessiliflorum Dunal) provenientes del Amazonas venezolano. Rev. Fac. Farm. 74(1), 41-45.

Rodríguez, D. B. 1997. Carotenoides y preparación de alimentos. USAID/DMNI Project. Instituto de Nutrición y Tecnología de Alimentos (INTA), Santiago de Chile.

Rojano, A., M. Botero, C. Monsalve y S. Ricaute. 2007. Capacidad reductora de 15 frutas tropicales. Scientia et Technica 8(33), 295-296.

Rojano, B. 1997. Oxidación de lípidos y antioxidantes. Departamento de Química, Universidad Nacional de Colombia, Medellín, Colombia. 
Singleton, V. y J. Rossi. 1965. Colorimetry of total phenolics with phosphotungstic acid reagents. Am. J. Enol. Viticult. 16, 114-158.

Sokol, R. 1997. Vitamina E. OPS. Conocimientos actuales sobre nutrición. ISLI, Washington D.C.

Soong, Y. y J.P. Barlow. 2004. Antioxidant activity and phenolic content of select fruit seeds. Food Chemistry 88, 411-417.

Soong, Y. y J.P. Barlow. 2005. Quantification of gallic and ellagic acid from longan (Dimocarpus longan Lour.) seed and mango (Mangifera indica L.) Kernel and their effects on antioxidant activity. Food Chem. 97, 524-530.

Stipanuk, M. 2000. Biochemical and physiological aspects of human nutrition. WB Scuenders Company, New York, NY.

Suaterna, A. 2001. Capacidad antioxidante total en extractos de frutas. Trabajo final. Especialización en Ciencia y Tecnología de Alimentos, Facultad de Ciencias Agropecuarias, Universidad Nacional de Colombia, Medellín, Colombia.
Takebayashi, J., T. Oki, J. Watanabe, K. Yamasaki, J. Chen, M.S. Furukawa, M.T. Utsugi, K. Taku, K. Goto, T. Matsumoto y Y. Ishimi. 2013. Hidrophilic antioxidant capacities of vegetables and fruits commoly consumed in japan and estimated average daily intake of hidropholic antioxidants from these foods. J. Food Comp. Anal. 29, 25-31.

Vasco, C., J. Ruales y A. Kamal-Eldin. 2008. Total phenolic compounds and antioxidant capacities of major fruits From Ecuador. Food Chem. 111(4), 816-823.

Velioglu, Y., G. Mazza, L. Gao y B. Oomah. 1998. Antioxidant activity and total phenolic in select fruits, vegetables and grain products. J. Agric. Food Chem. 46, 4113-4117.

Vinson, J.A., Y. Hao, X. Su y L. Zubik. 1998. Phenol antioxidant quality in foods: Vegetables. J. Agric. Food Chem. 46, 3630-3634.

Wang, S.Y. y H. Li. 2000. Antioxidant activity in fruit and leaves of blackberry, raspberry, and strawberry varies with cultivar and development stage. J. Agric. Food Chem. 45, 140-146. 